\title{
Diffusion and Current of Brownian Particles in Tilted Piecewise Linear Potentials: Amplification and Coherence
}

\author{
Els Heinsalu, Risto Tammelo*, Teet Örd \\ Institute of Theoretical Physics, Tartu University, 4 Tähe Street, 51010 Tartu, Estonia
}

\begin{abstract}
Overdamped motion of Brownian particles in tilted piecewise linear periodic potentials is considered. Explicit algebraic expressions for the diffusion coefficient, current, and coherence level of Brownian transport are derived. Their dependencies on temperature, tilting force, and the shape of the potential are analyzed. The necessary and sufficient conditions for the non-monotonic behavior of the diffusion coefficient as a function of temperature are determined. The diffusion coefficient and coherence level are found to be extremely sensitive to the asymmetry of the potential. It is established that at the values of the external force, for which the enhancement of diffusion is most rapid, the level of coherence has a wide plateau at low temperatures with the value of the Péclet factor 2. An interpretation of the amplification of diffusion in comparison with free thermal diffusion in terms of probability distribution is proposed.
\end{abstract}

PACS numbers: 05.40.-a, 05.60.-k, 02.50.Ey

\section{INTRODUCTION}

It is difficult to overestimate the importance of the effects caused by Brownian motion for soft condensed matter physics. An object of special attention has been Brownian motion in periodic structures which has various applications in condensed matter physics, chemical physics, nanotechnology, and molecular biology

[1, 2, 3, 4]. Furthermore, the idea that adding noise to deterministic motion can give nontrivial results has led to many important discoveries, such as stochastic resonance 5], resonant activation [6], noise-induced spatial patterns 7], noise-induced multistability as well as noise-induced phase transitions [3, 8, 9, 10, 11], ratchets [2], and hypersensitive transport [12], to name but a few of the new phenomena in this field.

The acceleration of thermal diffusion of Brownian particles in tilted periodic potentials has been studied recently in a number of papers [13, 14, 15, 16]. An small enhancement of diffusion induced by bias with respect to free diffusion was established for the asymptotic limits [13. The papers [14, 15, 16] provided exact analytic treatments of the effect for arbitrary temperature, tilting force, and periodic potential. A giant amplification of diffusion up to fourteen orders of magnitude was predicted in Refs. 14, 15]. Another new effect, a non-monotonic behavior of the diffusion coefficient and coherence level of the transport of Brownian particles as a function of temperature was found in Ref. 16]. Similar anomalies were observed in systems with spatially periodic temperature [17]. Recently it was shown that non-homogeneous dissipation can induce enhancement and suppression of the diffusion as a function of temperature, as well as an increase of the coherence level of Brownian motion in tilted symmetric periodic potential [18]. A distinctive behavior of diffusion also occurs if one applies a sinusoidal time-periodic force to the system, in addition to a tilted sinusoidal space-periodic force. It was demonstrated numerically that the interplay between frequency-locking and noise gives rise to a multi-enhancement of the effective diffusion, and a rich behavior of the current, including partial suppression and characteristic resonances 19. (see also [20]).

Usually, when addressing diffusion enhancement, tilted harmonic spatial periodic potential is used, confer however Ref. 16]. Furthermore, it is known that some features of the ratchet transport mechanism (e.g., the current reversals) are extremely sensitive to the shape of the potential [21]. In the present paper, we carry out a comprehensive study of the dependence of diffusive and coherent motion of overdamped Brownian particles on temperature and tilting force for various shapes of tilted piecewise linear periodic potentials. The latter are sufficiently simple to allow an algebraic treatment of the relevant quantities, being at the same time physically rich enough to provide most of the effects characteristic of tilted periodic potentials with one minimum per period.

The paper is organized as follows. Proceeding from the general scheme developed in Refs. 14, 15] (outlined in Sec. II), we derive in Sec. III the algebraic expressions for the diffusion coefficient, current, and Péclet factor for the case of a general tilted piecewise linear periodic potential. The asymptotic limits and particular cases are considered analytically in Sec. IV. In Sec. V we analyze the behavior of the diffusion coefficient and Péclet factor in the space of system parameters, i. e., the tilting force, temperature, and asymmetry parameter of the potential. We also discuss the effect of the amplification of diffusion in terms of probability distribution. The existence of correlation between the enhancement of diffusion and the stabilization of the coherence level of Brownian transport is demonstrated in Sec. VI. Our results are summarized in Sec. VII. 


\section{GENERAL SCHEME}

We consider overdamped motion of Brownian particles described by one-dimensional Langevin equation

$$
\eta \frac{\mathrm{d} x(t)}{\mathrm{d} t}=-\frac{\mathrm{d} V(x)}{\mathrm{d} x}+\xi(t),
$$

where $V(x)=V_{0}(x)-F x$ and $V_{0}(x)=V_{0}(x+L)$ is the periodic potential, $F$ is the static tilting force, $\eta$ is the coefficient of viscous friction, $\xi(t)$ is the Gaussian white noise with the mean value $\langle\xi(t)\rangle=0$ and the correlation function $\left\langle\xi(t) \xi\left(t^{\prime}\right)\right\rangle=2 \eta k_{B} T \delta\left(t-t^{\prime}\right)$.

As usual, the effective diffusion coefficient is defined by

$$
D=\lim _{t \rightarrow \infty} \frac{\left\langle x^{2}(t)\right\rangle-\langle x(t)\rangle^{2}}{2 t} .
$$

In the same time-scale the current is determined as

$$
\langle\dot{x}\rangle=\lim _{t \rightarrow \infty} \frac{\langle x(t)\rangle}{t} .
$$

According to Refs. 14, 15 the diffusion coefficient for the model (II) is equal to $(F \geq 0)$

$$
D=\frac{D_{0}}{N^{3}} \int_{x_{0}}^{x_{0}+L} I_{+}(x)\left[I_{-}(x)\right]^{2} \frac{\mathrm{d} x}{L},
$$

with

$$
\begin{gathered}
N=\int_{x_{0}}^{x_{0}+L} I_{-}(x) \frac{\mathrm{d} x}{L}, \\
I_{ \pm}(x)=\mp D_{0}^{-1} e^{ \pm V(x) / k_{B} T} \int_{x}^{x \mp L} e^{\mp V(y) / k_{B} T} \mathrm{~d} y .
\end{gathered}
$$

Here $x_{0}$ is an arbitrary point and $D_{0}=k_{B} T / \eta$ is the diffusion coefficient for $V_{0}(x)=0$, i.e., for free diffusion. The particle current is given by [14, 15, 22.

$$
\langle\dot{x}\rangle=N^{-1}\left(1-e^{-L F / k_{B} T}\right) .
$$

The relationship between the directed and diffusive components in Brownian motion (the level of coherence) can be characterized by the Péclet number $P e$ or by the factor of randomness $Q$

$$
P e=\frac{L\langle\dot{x}\rangle}{D}=\frac{2}{Q} .
$$

The greater the Péclet number, the greater the coherence of Brownian transport.

\section{ANALYTIC COMPUTATIONS}

We proceed form the exact analytical formulas for the effective diffusion coefficient (4) and current (7) of Brownian particles, and consider a piecewise linear periodic potential with the amplitude $A$, period $L$, and asymmetry parameter $k(0<k<L$; the potential is symmetric if $k=L / 2$ ). If the tilting force exceeds the critical value

$$
F>F_{c}=\frac{A}{L-k},
$$

the potential is monotonically decreasing, i.e., the potential does not have local minima.

We can take with no loss of generality $L=1$ and replace the relevant quantities with the corresponding dimensionless ones: $\tilde{T}=k_{B} T A^{-1}$ and $\tilde{F}=F / F_{c}$, hence $\tilde{F}_{c}=1, \tilde{D}=D \eta A^{-1}, \tilde{D}_{0}=D_{0} \eta A^{-1}$ so that $\tilde{D}_{0}=\tilde{T}$ and $\langle\tilde{\dot{x}}\rangle=\eta A^{-1}\langle\dot{x}\rangle$. For brevity, in what follows we will omit the tilde signs above the symbols.

Now the expressions for the diffusion coefficient, current, and Péclet factor have the following form:

$$
\langle\dot{x}\rangle=\varphi_{0} Z^{-1},
$$

$$
D=T Y Z^{-3}
$$

$$
P e=\varphi_{0} Z^{2}(T Y)^{-1}
$$

where

$$
\begin{gathered}
\varphi_{0}=1-\exp \left(-\frac{F}{T(1-k)}\right), \\
Z=\int_{0}^{k} H_{-a}(x) \mathrm{d} x+\int_{k}^{1} H_{-b}(x) \mathrm{d} x, \\
Y=\int_{0}^{k} H_{+a}(x)\left[H_{-a}(x)\right]^{2} \mathrm{~d} x \\
+\int_{k}^{1} H_{+b}(x)\left[H_{-b}(x)\right]^{2} \mathrm{~d} x .
\end{gathered}
$$

Equations (14) and (15) contain the functions $H_{ \pm a}$ and $H_{ \pm b}$ where the subscripts $a$ and $b$ associate, correspondingly, with the limits of integration from 0 to $k$ and from $k$ to 1 . We have 


$$
\begin{aligned}
& H_{ \pm a}(x)=D_{0}^{-1} e^{\left[ \pm v_{a}(x)-F(1 \pm 1) / 2(1-k)\right] / T}\left\{\int_{x}^{k} e^{\mp v_{a}(y) / T} \mathrm{~d} y+\int_{k}^{1} e^{\mp v_{b}(y) / T} \mathrm{~d} y+\int_{1}^{x+1} e^{\mp v_{\tilde{a}}(y) / T} \mathrm{~d} y\right\} \\
& H_{ \pm b}(x)=D_{0}^{-1} e^{\left[ \pm v_{b}(x)-F(1 \pm 1) / 2(1-k)\right] / T}\left\{\int_{x}^{1} e^{\mp v_{b}(y) / T} \mathrm{~d} y+\int_{1}^{k+1} e^{\mp v_{\tilde{a}}(y) / T} \mathrm{~d} y+\int_{k+1}^{x+1} e^{\mp v_{\tilde{b}}(y) / T} \mathrm{~d} y\right\}
\end{aligned}
$$

In Eqs. (16) the dimensionless potential reads as

$$
\begin{aligned}
& v_{a}(x)=a_{0}-a x, \quad 0 \leq x \leq k, \\
& v_{b}(x)=-b_{0}+b x, \quad k \leq x \leq 1, \\
& v_{\tilde{a}}(x)=\tilde{a}_{0}-a x, \quad 1 \leq x \leq 1+k, \\
& v_{\tilde{b}}(x)=-\tilde{b}_{0}+b x, \quad 1+k \leq x \leq 2,
\end{aligned}
$$

with

$$
\begin{aligned}
& a=\frac{1-(1-F) k}{(1-k) k}, \quad a_{0}=1, \quad \tilde{a}_{0}=\frac{1+k}{k}, \\
& b=\frac{1-F}{1-k}, \quad b_{0}=\frac{k}{1-k}, \quad \tilde{b}_{0}=\frac{1+k}{1-k} .
\end{aligned}
$$

Performing integration in Eqs. (16), we obtain

$$
\begin{aligned}
H_{ \pm a}(x)= & \frac{\varphi_{0}}{a}+g \varphi_{a} \\
& \times \exp \left(\frac{a[\mp 2 x-k(1 \mp 1)]}{2 T}\right), \\
H_{ \pm b}(x)= & -\frac{\varphi_{0}}{b}+g \varphi_{b} \\
& \times \exp \left(\frac{b[ \pm 2(x-1)+(1-k)(1 \pm 1)]}{2 T}\right),
\end{aligned}
$$

with the notations

$$
\begin{gathered}
g=\frac{1}{a}+\frac{1}{b}, \\
\varphi_{a}=\exp \left(\frac{1-F}{T}\right)-1, \\
\varphi_{b}=1-\exp \left(-\frac{1-(1-F) k}{T(1-k)}\right) .
\end{gathered}
$$

Substituting the functions $H_{ \pm a, b}(x)$ from Eqs. (19) into (14) and (15), we have after integration

$$
Z=\left(\frac{k}{a}-\frac{1-k}{b}\right) \varphi_{0}+T g^{2} \varphi_{a} \varphi_{b}
$$

$$
\begin{aligned}
Y= & \left(\frac{k}{a^{3}}-\frac{1-k}{b^{3}}\right) \varphi_{0}^{3}+3 T\left(\frac{1}{a^{3}}+\frac{1}{b^{3}}\right) g \varphi_{0}^{2} \varphi_{a} \varphi_{b} \\
& +\frac{1}{2} T g^{2} \varphi_{0}\left[\frac{1}{a^{2}} \varphi_{a}^{2} \tilde{\varphi_{b}}-\frac{1}{b^{2}} \varphi_{b}^{2} \tilde{\varphi_{a}}\right] \\
& +2 g^{2} \varphi_{0}\left[\frac{k}{a} \varphi_{a}^{2}\left(1-\varphi_{b}\right)-\frac{1-k}{b} \varphi_{b}^{2}\left(1+\varphi_{a}\right)\right] \\
& +T g^{3}\left[\frac{1}{a} \varphi_{a}^{3} \varphi_{b}\left(1-\varphi_{b}\right)+\frac{1}{b} \varphi_{b}^{3} \varphi_{a}\left(1+\varphi_{a}\right)\right]
\end{aligned}
$$

where

$$
\begin{aligned}
& \tilde{\varphi_{a}}=\exp \left(\frac{2(1-F)}{T}\right)-1, \\
& \tilde{\varphi_{b}}=1-\exp \left(-\frac{2[1-(1-F) k]}{T(1-k)}\right) .
\end{aligned}
$$

By that we have derived the exact algebraic expressions for the current $\langle\dot{x}\rangle$, the diffusion coefficient $D$, and the Péclet factor $P e$.

\section{ASYMPTOTIC LIMITS AND PARTICULAR CASES}

In this Section we will examine the asymptotic limits and essential particular cases on the basis of the analytical formulas derived.

(i) In the absence of tilt $(F=0)$, Eqs. (13) and (21) reduce to

$$
\varphi_{0}=0, \quad \varphi_{a}=e^{1 / T}-1, \quad \varphi_{b}=1-e^{-1 / T},
$$

and from Eqs. (11), (22), and (23) one obtains

$$
D=\frac{1}{2 T[\cosh (1 / T)-1]} .
$$

This expression is as a special case of the general formula of the diffusion coefficient for arbitrary unbiased periodic potential [23]. It is to be noticed, that for $F=0$ the coefficient of diffusion becomes independent of the asymmetry parameter $k$.

(ii) In the high temperature limit, one can take into account only the first order terms in the expansions of 
the exponents in Eqs. (13) and (21). Then

$$
\varphi_{0} \approx \frac{F}{T(1-k)}, \varphi_{a} \approx \frac{1-F}{T}, \varphi_{b} \approx \frac{1-(1-F) k}{T(1-k)}
$$

and

$$
H_{ \pm a, b}=T^{-1}, \quad Z=T^{-1}, \quad Y=T^{-3} .
$$

The current, diffusion coefficient, and Péclet factor now become

$$
\begin{gathered}
\langle\dot{x}\rangle=\frac{F}{1-k}, \\
D=T, \\
P e=\frac{F}{T(1-k)} .
\end{gathered}
$$

(iii) Under the conditions $F \gg 1$ and $F / T \gg 1$, it is valid that

$$
\varphi_{0} \approx-\varphi_{a} \approx \varphi_{b} \approx 1, \quad a \approx-b \approx \frac{F}{1-k},
$$

and

$$
H_{ \pm a, b}=\frac{1-k}{F}, \quad Z=\frac{1-k}{F}, \quad Y=\left(\frac{1-k}{F}\right)^{3} .
$$

As a result, the expressions for $D,\langle\dot{x}\rangle$, and $P e$ coincide with Eqs. (29)-31). Thus, at high temperatures and at large values of tilting force, the transport properties of Brownian particles are the same.

(iv) If $F<1$ and $(1-F) / T \gg 1$, we have the following asymptotic limits:

$$
\begin{aligned}
& \varphi_{b} \approx \tilde{\varphi}_{b} \approx 1, \\
& \varphi_{a} \approx e^{(1-F) / T} \gg 1, \\
& \tilde{\varphi}_{a} \approx e^{2(1-F) / T}=\varphi_{a}^{2} .
\end{aligned}
$$

Then Eqs. (14), 15), and (19) yield

$$
\begin{gathered}
Z=T g^{2} e^{(1-F) / T}, \\
Y=\frac{1}{2} T g^{3} e^{2(1-F) / T}\left[\left(a^{-1}-b^{-1}\right) \varphi_{0}\right. \\
\left.+2\left(a^{-1} e^{-F /(1-k) T}+b^{-1}\right)\right],
\end{gathered}
$$

and

$$
\begin{aligned}
& \langle\dot{x}\rangle=\frac{\varphi_{0}}{T g^{2} e^{(1-F) / T}}, \\
& D=\frac{2-\varphi_{0}}{2 T g^{2} e^{(1-F) / T}},
\end{aligned}
$$

$$
P e=\frac{2 \varphi_{0}}{2-\varphi_{0}}=2 \tanh \frac{F}{2 T(1-k)}
$$

(cf. also [16]). If, additionally, the condition $F / T(1-k) \gg 1$ is fulfilled, it is valid that $e^{-F / T(1-k)} \approx 0$ and $\varphi_{0} \approx 1$. Consequently, in the present case we have $2 D=\langle\dot{x}\rangle$ and $P e=2$. This indicates that an extremely exact stabilization of the level of coherence of Brownian transport occurs in this region of parameters.

(v) At the critical tilt $(F=1)$, it is valid that

$$
\begin{aligned}
H_{ \pm a}(x)= & \frac{\varphi_{0}}{a}+\frac{1-k}{T} \exp \left(\frac{a[\mp 2 x-k(1 \mp 1)]}{2 T}\right) \\
H_{ \pm b}(x)= & \frac{\varphi_{0}}{a}+\frac{1-k}{T} \exp \left(-\frac{a k}{T}\right) \\
& +\frac{\varphi_{0}[ \pm 2(x-1)+(1-k)(1 \pm 1)]}{2 T}
\end{aligned}
$$

whereas in the low-temperature limit Eqs. (10)-(15) and (40) yield

$$
\begin{gathered}
\langle\dot{x}\rangle=\frac{2 T}{(1-k)^{2}}, \\
D=\frac{2 T}{3(1-k)^{2}}, \\
P e=3 .
\end{gathered}
$$

We observe that, for $F=1$ the Péclet factor is constant and depends neither on the temperature nor on the asymmetry parameter.

\section{NON-MONOTONIC BEHAVIOR OF DIFFUSION AND COHERENCE}

The expression of the diffusion coefficient as a function of $F$ given by Eqs. (11), (22), and (23) reveals a qualitatively similar behavior to that found in Refs. [12,13], exhibiting a resonant-like maximum if the temperature is sufficiently low. This effect is strongly influenced by the shape of the periodic potential, as illustrated in Fig. 1 This plot shows that for positive bias $(F>0)$, the increase of the value of $k$ favors the amplification of diffusion compared to free thermal diffusion.

Next we consider the dependence of diffusion on temperature. Figure 2 displays the typical behavior of the diffusion coefficient as a function of temperature and asymmetry parameter for $F=0.95$. The non-monotonic behavior occurs if the value of tilting force lies within a certain range $F_{1}<F<F_{2}$ where $F_{1,2}$ depend on $k$. One can see that the maximum of $D(T)$ becomes rapidly narrower and higher as $k$ approaches unity. There exist the limiting values $k_{E} \approx 0.8285$ and $F_{0} \approx 1.1292$ : for $k<k_{E}$ 


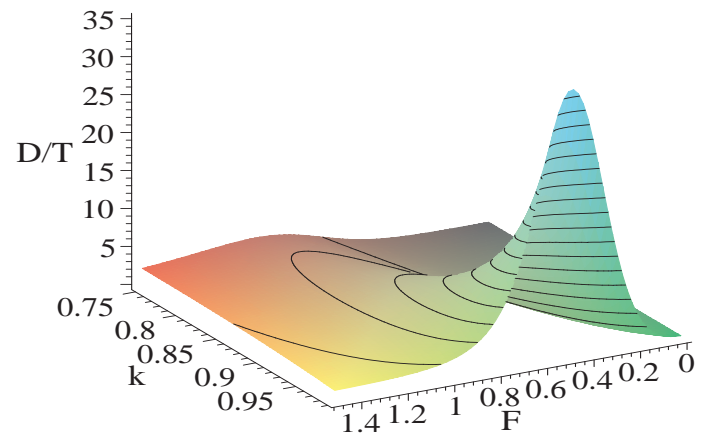

FIG. 1: The plot of the diffusion coefficient $v s$ the tilting force and the potential asymmetry parameter at fixed $T=0.1$.

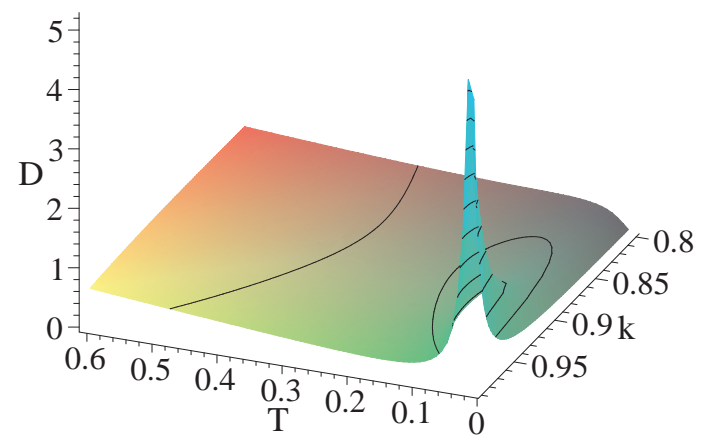

FIG. 2: The plot of the diffusion coefficient $v s$ the temperature and the potential asymmetry parameter at fixed $F=0.95$.

or $F>F_{0}$ the diffusion coefficient is a monotonic function of temperature for all values of bias and asymmetry coefficient. Therefore, the $(k, F)$-space is divided into two domains where the analytical properties of the diffusion coefficient as a function of temperature are qualitatively different.

The curves of the Péclet factor versus temperature are depicted in Fig. 3. The function $P e(T)$ passes through a maximum (curves 1-5) for $F<F_{c}$, which is also present slightly above the critical tilt (curve 6). With a further increase of $F$, the maximum of $P e(T)$ disappears. As seen in Fig. 3, the optimal level of Brownian transport, determined by the maximal value of the Péclet number, is sensitive to the shape of periodic potential: at $F<F_{c}$ the optimal level of transport rises with an increase in $k$. At the same time, the larger values of $k$ make a minimum of $D(T)$, which follows a maximum of $D(T)$ at a higher value of temperature, deeper (the effect can be anticipated in Fig. 2). In this sense the situation is analogous to the results of Ref. [18], where the enhancement of the coherence of Brownian motion in a certain region of temperature due to frictional inhomogeneity is associated with the suppression of diffusion by the same factor.

Now we discuss the amplification of diffusion by bias in terms of probability distribution. The stationary probability density for the coordinate of a Brownian particle

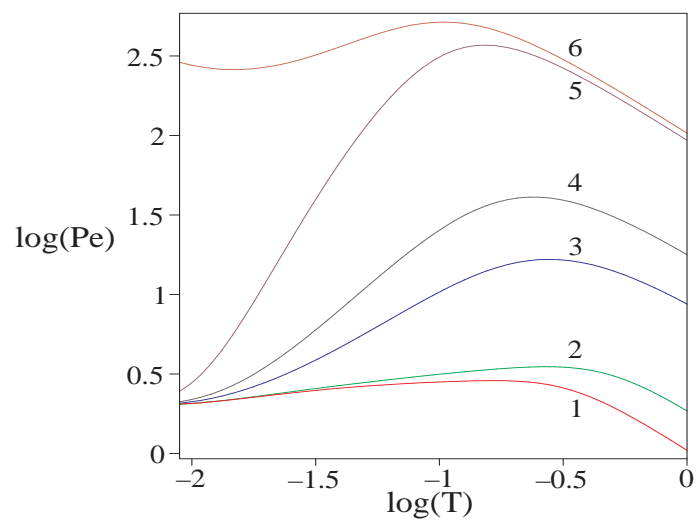

FIG. 3: The plot of the Péclet factor $\log _{10}(P e)$ versus temperature $\log _{10}(T)$ for various values of the potential asymmetry coefficient at $F=0.95$ (curves $1-5$ ) and $F=1.05$ (curve 6). Curve 1: $k=0.1$, curve 2: $k=0.5$, curve $3: k=0.9$, curve 4 : $k=0.95$, curves $5,6: k=0.99$.

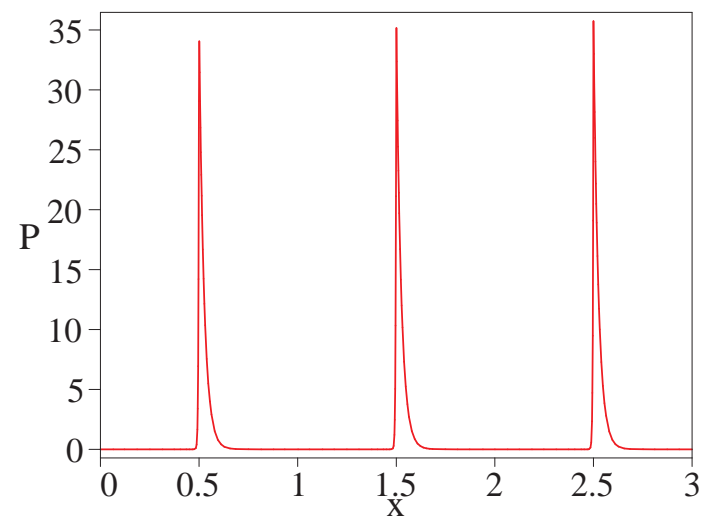

FIG. 4: The plot of the probability density $P(x)$ at $T=0.01$, $k=0.5$ and $F=0.8$.

[1] normalized over one period can be written as

$$
P(x)=\frac{1}{N} e^{-V(x) / k_{B} T} \int_{x}^{x+L} e^{V(y) / k_{B} T} \mathrm{~d} y .
$$

Figures 4-6 represent (in terms of the dimensionless parameters) the probability distributions characteristic to various diffusion levels depending on the tilting force.

Figure 4 illustrates the situation, where particles are mainly localized around the minima of the potential and transport is strongly suppressed. Diffusion is essentially weaker in comparison with free diffusion: $D / T \sim 10^{-6}$. The probability distribution shown in Fig. 5 corresponds to the case where the diffusion is approximately maximal $(D / T=3.5)$ for the chosen values of temperature and asymmetry coefficient. In this case the regions with a large probability are separated by the domains where the probability is much smaller, however, large enough to allow the entrance of a sufficient number of particles into these domains. As a result, a channel of hopping-like 


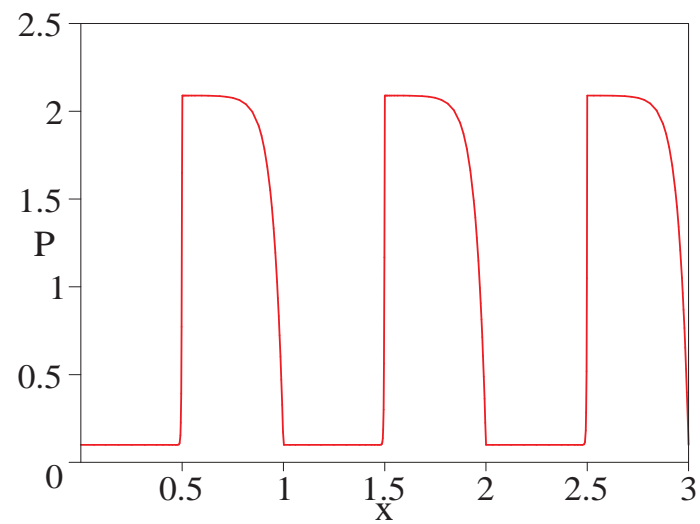

FIG. 5: The plot of the probability density $P(x)$ at $T=0.01$, $k=0.5$ and $F=1.1$.

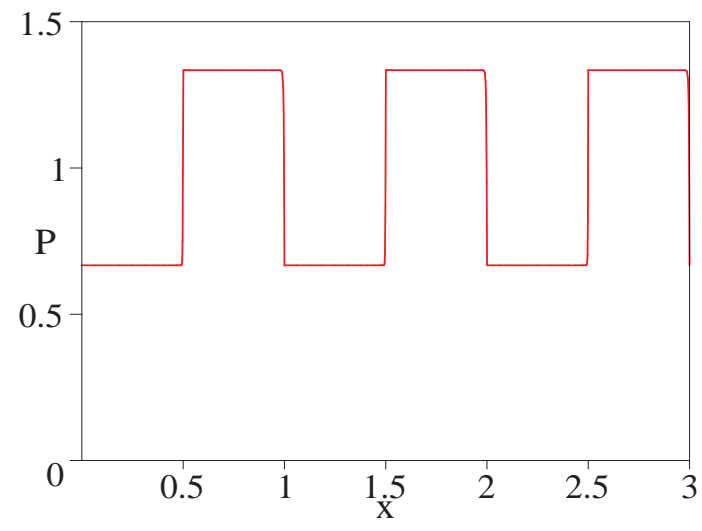

FIG. 6: The plot of the probability density $P(x)$ at $T=0.01$, $k=0.5$ and $F=3$.

transport is formed, leading to the enhancement of diffusion with respect to free diffusion. The further increase of the tilting force $F$ makes the probability distribution still more homogeneous, as seen in Fig. 6, and the diffusion approaches the free diffusion limit $(D / T=1.3$ for the values of the parameters used in Fig. 6).

Consequently, the amplified diffusion in the tilted periodic potential is characterized by the specific inhomogeneous probability distribution with spatially alternating domains of high and low probability. The occurrence of a maximum in the dependence $D(T)$ can be understood in a similar way.

\section{CORRELATION BETWEEN THE ENHANCEMENT OF DIFFUSION AND STABILIZATION OF COHERENCE}

With regard to the simultaneous enhancement of diffusion and current, caused by the force $F$, with respect to an untilted system, the relation between $D$ and $\langle\dot{x}\rangle$ is of interest. One can expect that such a relationship reflects some intrinsic features of the mutual influence between

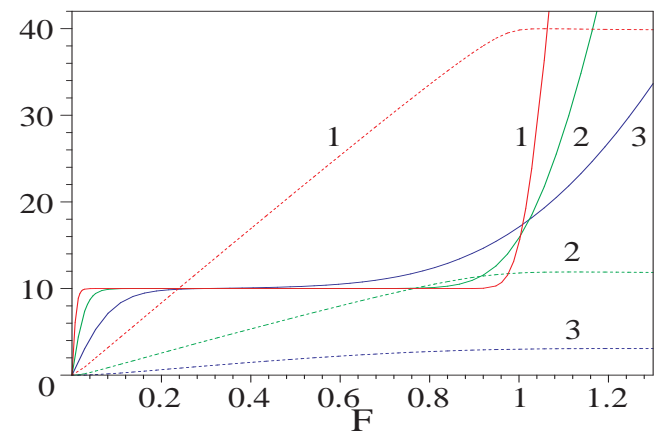

FIG. 7: The comparison of the dependencies of the Péclet factor and diffusion coefficient on the tilting force for various temperatures at fixed $k=0.5$. Solid lines: $5 \times P e$ vs $F$, dashed lines: $\log _{10}[D(F) / D(0)]$ vs $F$. Curves $1: T=0.01$, curves 2 : $T=0.03$, curves 3: $T=0.09$.

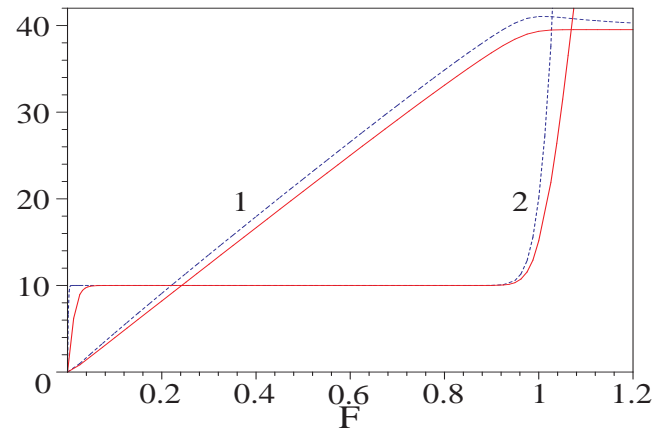

FIG. 8: The comparison of the dependencies of the Péclet factor and diffusion coefficient on the tilting force for $k=0.1$ (solid lines) and $k=0.9$ (dashed lines) at fixed $T=0.01$. Curves 1: $\log _{10}[D(F) / D(0)]$ vs $F$, curves $2: 5 \times$ Pe vs $F$.

diffusion and current driven by the tilt merely at lower temperatures, when the initial suppression of both components of Brownian transport by periodic potential is stronger.

The comparative plot of $D$ and Pe versus $F$ is presented in Figs. 7 and 8. One can see that the function $\mathrm{Pe}(F)$ has a point of inflection which turns into a wide plateau at lower temperatures. For values of $F$, from zero up to the end of the plateau, the behavior of $\operatorname{Pe}(F)$ is described with great accuracy by Eq. (39). Note especially that the domain where $P e=2$ coincides with the domain where the increase of diffusion coefficient as a function of $F$ is most rapid and follows with a good approximation the law $D=c^{\alpha_{1}+\alpha_{2} F}$, where $c$ and $\alpha_{1,2}$ depend on $T$ and $k$. The location of the end of this region at larger values of $F$ is quite insensitive to the shape of the periodic potential, as seen in Fig. 8, and is located approximately at $F_{c}$. 


\section{CONCLUSION}

In the present work we have addressed the problem of overdamped motion of Brownian particles on tilted piecewise linear potentials in the presence of white thermal noise. We have derived the exact algebraic expressions for the diffusion coefficient, particle current, and factor of randomness, which are valid for an arbitrary simple sawtooth potential, tilting force, and strength of the thermal noise. On the basis of these formulas we have discussed the characteristic limiting cases and asymptotic behavior.

Acceleration of diffusion turns out to be very sensitive to the shape of the piecewise linear potential. It is shown that large values of the asymmetry parameter $k$ favor the amplification of diffusion by means of biased potential and temperature in comparison with free thermal diffusion. This can be understood as a result of the formation of probability distribution with spatially alternating regions of specifically balanced high and low probability. The necessary and sufficient conditions for the non-monotonic behavior of the diffusion coefficient as a function of temperature are established. On the basis of these results, among the other applications, one can expect the significant increase of diffusive transport, for example, in the superionic conductors 24, 25] in a strong external electric field.

We have demonstrated that the Péclet factor as a function of the tilting force has a plateau at low temperatures which terminates with a steep rise at the critical value of the tilting force. As temperature grows, the plateau gradually reduces until disappears and the Péclet factor becomes monotonically increasing. The domain, where the Péclet factor exhibits the plateau, coincides with the domain where the enhancement of diffusion coefficient is maximal. Consequently, in the region of parameters where substantial acceleration of diffusion occurs, the current and diffusion are exactly synchronized. Note that the stabilization of the coherence level of Brownian transport takes place at the value of the Péclet factor $P e=2$ which is also characteristic for Poisson processes 1], such as the Poisson enzymes in kinesin kinetics [26, 27, 28].

Finally, it is remarkable that, as a result of the interplay of periodic potential, bias and white noise, there exists an exact correlation between the acceleration of diffusion, induced by the tilting force, and the stabilization of the coherence level of Brownian motion. It seems that this phenomenon is quite universal and manifests itself for an arbitrary periodic potential in situations where initially strongly suppressed transport is enhanced by bias, which generates significant amplification of diffusion in comparison with free diffusion.

\section{Acknowledgement}

The authors are grateful to Romi Mankin and Marco Patriarca for valuable discussions, and acknowledge support by Estonian Science Foundation through Grant No. 5662 .
[*] E-mail address: tammelo@ut.ee

[1] H. Risken, The Fokker-Planck Equation (SpringerVerlag, Berlin, 1996).

[2] P. Reimann, Phys. Rep. 361, 57 (2002).

[3] P. S. Landa and P. V. E. McClintock, Phys. Rep. 323, 1 (2000).

[4] O. M. Braun and Yu. S. Kivshar, Phys. Rep. 306, 1 (1998).

[5] L. Gammaitoni, P. Hänggi, P. Jung, and F. Marchesoni, Rev. Mod. Phys. 70, 223 (1998).

[6] C. R. Doering and J. C. Gadoua, Phys. Rev. Lett. 69, 2318 (1992).

[7] J. Garcia-Ojalvo, A. Hernandez-Machado, and J. M. Sancho, Phys. Rev. Lett. 71, 1542 (1993).

[8] C. Van den Broeck, J. M. R. Parrondo, and R. Toral, Phys. Rev. Lett. 73, 3395 (1994).

[9] C. Van den Broeck, J. M. R. Parrondo, R. Toral, and R. Kawai, Phys. Rev. E 55, 4084 (1997).

[10] P. S. Landa, Nonlinear Oscillations and waves in dynamical systems (Kluwer, Dordrecht, 1996).

[11] J. Garcia-Ojalvo and J. M. Sancho, Noise in spatially extended systems (Springer, Berlin, 1999).

[12] S. L. Ginzburg and M. A. Pustovoit, Phys. Rev. Lett. 80, 4840 (1998).

[13] G. Constantini and F. Marchesoni, Europhys. Lett. 48, 491 (1999).

[14] P. Reimann, C. Van den Broeck, H. Linke, P. Hänggi,
J. M. Rubi, and A. Pérez-Madrid, Phys. Rev. Lett. 87, 010602 (2001).

[15] P. Reimann, C. Van den Broeck, H. Linke, P. Hänggi, J. M. Rubi, and A. Pérez-Madrid, Phys. Rev. E 65, 031104 (2002).

[16] B. Lindner, M. Kostur, and L. Schimansky-Geier, Fluct. Noise Lett. 1, R25 (2001).

[17] B. Lindner and L. Schimansky-Geier, Phys. Rev. Lett. 89, 230602 (2002).

[18] D. Dan and A. M. Jayannavar, Phys. Rev. E 66, 041106 (2002).

[19] D. Reguera, P. Reimann, P. Hänggi, and J. M. Rubi, Europhys. Lett. 57, 644 (2002).

[20] H. Gang, A. Daffertshofer and H. Haken, Phys. Rev. Lett. 76, 4874 (1996).

[21] R. Mankin, A. Ainsaar, and E. Reiter, Phys.Rev. E 61 6359 (2000); R. Mankin, A. Ainsaar, A. Haljas, and E. Reiter, ibid. 63041110 (2001); R. Mankin, R. Tammelo, and D. Martila, ibid. 64, 051114 (2001); R. Tammelo, R. Mankin, and D. Martila, ibid. 66, 051101 (2002); R. Mankin, A. Haljas, R. Tammelo, and D. Martila, ibid. 68, 011105 (2003).

[22] R. L. Stratonovich, Radiotekh. Elektron. 3, 497 (1958).

[23] S. Lifson and J. L. Jackson, J. Chem. Phys. 36, 2410 (1962).

[24] M. B. Salamon (Ed.), The Physics of Superionic Conductors (Springer-Verlag, Berlin, Heidelberg, 1979). 
[25] J. W. Perram (Ed.), The Physics of Superionic Conductors and Electrode Materials (Plenum Press, New York, 1983).

[26] K. Svoboda, P. P. Mitra, and S. M. Block, Proc. Natl. Acad. Sci. USA 91, 11782 (1994).
[27] M. J. Schnitzer and S. M. Block, Nature 388, 386 (1997).

[28] K. Visscher, M. J. Schnitzer, and S. M. Block, Nature 400, 184 (1999). 